\title{
Sole acquired trisomy 21 in a case of CD7 and CD10 positive acute myeloid leukemia
}

Sir,

A 52-year-old lady presented with fever and pallor since two months. She had bilateral cervical lymph node enlargement, gum hypertrophy, subconjunctival hemorrhage and sternal tenderness. Liver was enlarged 3 $\mathrm{cm}$ below costal margin. Spleen was not palpable. Her hemoglobin $(\mathrm{H} \mathrm{b}$ ) $83 \mathrm{~g} / \mathrm{L}$, total leukocyte count (TLC) $138.9 \times 10 \%$ L and platelet count was $31 \times 10^{\%} / \mathrm{L}$. Peripheral smear showed $96 \%$ blasts. The bone marrow aspirate showed a hypercellular marrow with $96 \%$ blasts with auer rods. These blasts were myeloperoxidase, sudan black and non-specific esterase positive. These blasts were CD 33 and HLA DR positive. There was a co expression of CD 7 and CD 10.

The 48h unstimulated culture-GTG banding demonstrated $47, X X,+21$ in $100 \%$ cells examined [Figure 1]. There were no signs of Down's syndrome in this lady.

Fluorescent in situ hybridization (FISH) done on buccal smears using the probe LSI 21 for 21q22.13-q22.2region spectrum orange (Vysis, USA) showed that there was no aneuploidy of Chromosome 21 [Figure 2]. This was done to rule out congenital mosaic. FISH was negative for inv (16) (p13q22) \& t $(16 ; 16)(p 13 q 22)$. Thus a final diagnosis of Acute myeloid leukemia (AML-M 4) with sole acquired +21 was made.

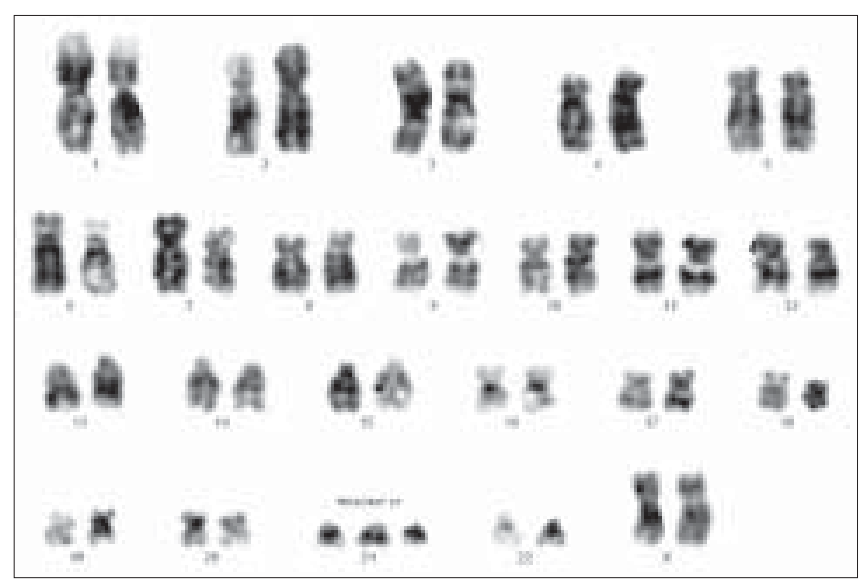

Figure 1: Karyotype of the patient showing 47, XX, +21 
Table 1: Cases of acute myeloid leukemia and sole acquired +21

\begin{tabular}{|c|c|c|c|c|c|}
\hline Reference & Age (years) & Sex & AML subtype & CD7 & Outcome \\
\hline \multirow[t]{2}{*}{ Wan et al. ${ }^{[1]}$} & 28 & M & M 2 & NA & No treatment \\
\hline & 78 & $\mathrm{~F}$ & M 4 & NA & $\mathrm{CR}$ \\
\hline Kondo et al. ${ }^{[2]}$ & 21 & M & M 2 & + & $\mathrm{CR}$, well at 4 months \\
\hline Yamamoto et al. ${ }^{[3]}$ & 49 & M & M 2 & + & $\begin{array}{l}\text { CR, MDS } 2 \text { years later, leukemia } 3 \text { years } \\
\text { later, died at } 4 \text { years }\end{array}$ \\
\hline Udayakumar et al. ${ }^{[4]}$ & 24 & M & M 2 & + & $\mathrm{CR}$ \\
\hline \multirow[t]{3}{*}{ Wei et al. ${ }^{[1]}$} & 35 & M & M 4 & NA & NA \\
\hline & 30 & M & NA & NA & - \\
\hline & 28 & $\mathrm{~F}$ & M 2 & NA & - \\
\hline Present case & 52 & $\mathrm{~F}$ & M 4 & + & CR, well at 2 months \\
\hline
\end{tabular}

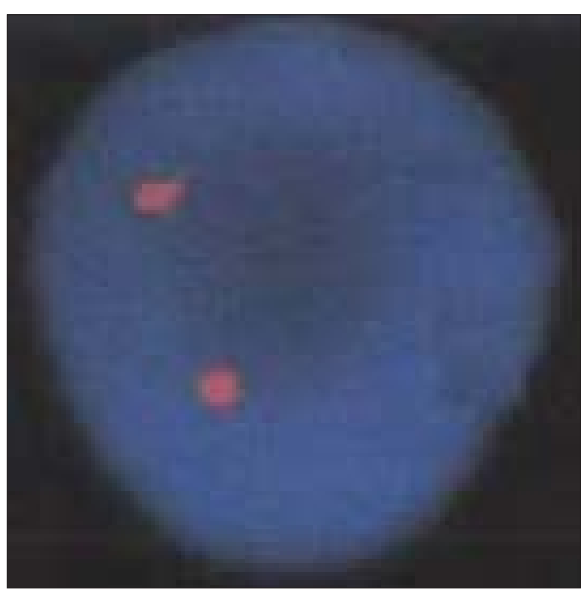

Figure 2: Fluorescent in situ hybridization on buccal smears for 21q22.13-q22.2-region shows no aneuploidy of chromosome 21

She was given standard $3+7$ chemotherapy. $\mathrm{H}$ er neutrophils recovered on day 27 and platelet counts recovered on day 37. A bone marrow aspirate done on day 36 showed her disease to be in remission. She was then given consolidation therapy with high dose cytarabine. Dose was not reduced and she tolerated it well.

Trisomy $21(+21)$ in $A M L$ is usually present in conjunction with other cytogenetic changes, whose presence rather than +21 determines the clinical outcome. The incidence of +21 as a sole abnormality was between $0.3 \%$ in all patients with $A M L . .^{[1-4]}$ Morphologically, AM L with +21 as a sole abnormality preferentially shows $M 2$ or M 4 phenotypes according to the FAB classification. ${ }^{[1-4]}$ Cells trisomic for Chromosome 21 could be over-proliferating due to enhanced expression of a tumourigenic protein coded by a Chromosome 21 genes. $^{[1]}$

Expression of lymphoid antigens is common in $A M L$. CD 2 is expressed in $16-21 \%$ and CD 19 in $7-14 \%$.
Co expression of CD 7 on leukemic blasts has been documented in approximately $15 \%$ of $A M L .^{[2]} \mathrm{A}$ high incidence of co-expression of CD 7 has been documented in the overall Down syndrome patients with leukemia. $C D 10$ has been occasionally reported in $A M L^{5}$. The prognostic value of $C D 7$ and $C D 10$ expression in $A M L$, however, is unclear. $C D 7+A M L$ patients have a significantly lower response rate and poorer prognosis than CD 7- AML patients [Table 1]..$^{[5]}$

Similarly, AML patients with acquired +21 as sole abnormality have been considered to have a poor prognosis. ${ }^{[1]} \mathrm{H}$ owever, patients have shown $\mathrm{CR}$ and good prognostic indication. ${ }^{[2,3]}$ Co-expression of CD 7 is probably indicative of the very early stage at which the cell became malignant. ${ }^{[4]}$

We conclude that sole acquired +21 with co-expression of $C D 7$ in $A M L$ is a rare phenomenon. Further data is required to assess the prognostic significance of $C D$ 7 in this subgroup.

\section{Naithani R, Singhal D, Kumar R, Raina V',} Saxena R, Anand H

Department of Hematology, All India Institute of Medical Sciences, New Delhi, India. 'Department of Immunology \& Molecular biology, Indraprastha Apollo Hospital, New Delhi, India.

Correspondence to: Dr. Rahul Naithani, E-mail:dr_rahul6@hotmail.com

\section{References}

1. Wan TS, Au WY, Chan JC, Chan LC, Ma SK. Trisomy 21 as the sole acquired karyotypic abnormality in acute myeloid leukemia and myelodysplastic syndrome. Leuk Res 1999;23:1079-83.

2. Kondo H, Kobayashi A, Iwasaki H. Trisomy 21 as the sole acquired karyotypic abnormality in an adult patient with CD7-positive acute 
myeloid leukemia. Cancer Genet Cytogenet 2001;127:77-9.

3. Yamamoto K, Nagata K, Hamaguchi H. A new case of CD7-positive acute myeloblastic leukemia with trisomy 21 as a sole acquired abnormality. Cancer Genet Cytogenet 2002; 133: 183-4.

4. Udayakumar AM, Pathare AV, Muralitharan S, Alghzaly AA, Alkindi $S$, Raeburn JA. Trisomy 21 as a sole acquired abnormality in an adult Omani patient with CD7- and CD9-positive acute myeloid leukemia. Arch Med Res 2007;38:797-802.

5. Del Poeta G, Stasi R, Venditti A, Suppo G, Aronica G, Bruno A, et al. Prognostic value of cell marker analysis in de novo acute myeloid leukemia. Leukemia. 1994;8:388-94. 